\section{Navicular stress fracture}

\section{R V P de Villiers MMed (RadD) SA}

Department of Radiology, Tygerberg Academic Hospital and University of Stellenbosch, Tygerberg, W Cape

\section{Abstract}

The diagnosis of a navicular fracture is difficult and often delayed due to a nonspecific clinical presentation and subtle imaging findings. Initial radiographs are often negative. Historically isotope bone scans were recommended, but now $\mathrm{CT}$ and MR imaging are suggested due to the higher specificity. Imaging of both feet allows internal comparison and may also detect an asymptomatic or unsuspected contralateral fracture. $\mathrm{CT}$ is a reliable indicator of fracture healing. The imaging evidence of fracture healing usually lags behind the clinical picture.

\section{Introduction}

Navicular fractures commonly occur in elite athletes, including runners, gymnasts and football players.

\section{Clinical}

The diagnosis is often delayed for months. Clinically the onset is insidious with nonspecific signs and symptoms. The interval between symptoms and the diagnosis may be from 7 weeks to 4 months or longer.

Clinically the patient complains of pain along the dorsomedial aspect of the midfoot. On examination there may be pain to palpation along the medial longitudinal arch or dorsum of the foot. Foot anomalies that may be associated with navicular stress fractures are a short first metatarsal and a relatively long second ray.

\section{Pathology}

Most navicular stress fractures involve the middle third of the navicular. This is due to a relative avascularity of the middle third of the navicular. Fractures may be complete or incomplete. Incomplete fractures involve the dorsal $5 \mathrm{~mm}$ of the navicular adjacent to the talonavicular joint. $^{2}$

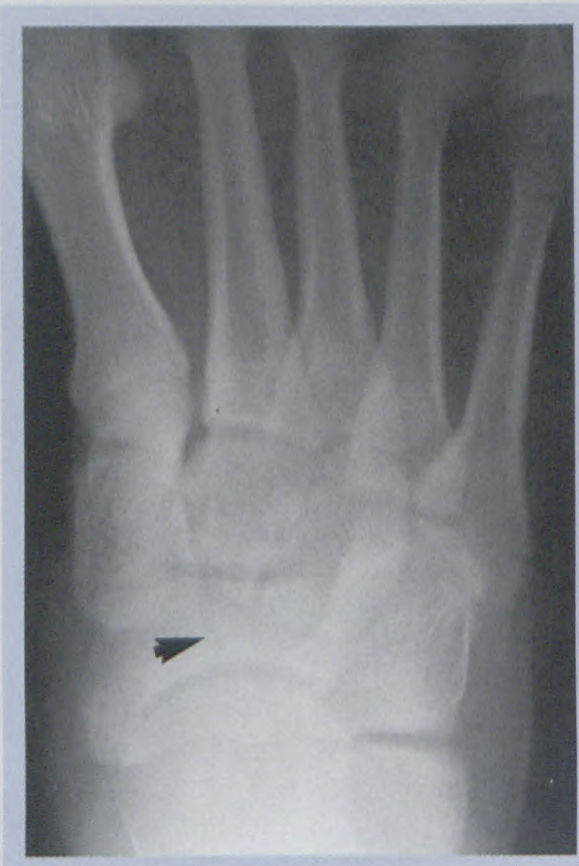

Fig. 1. Plain $X$-ray of subtle fracture of navicular bone.

\section{Imaging}

Imaging of a suspected stress fractures starts with radiographs of the foot. The initial radiographs are often negative (Fig. 1). Historically isotope bone scans were recommended, but now $\mathrm{CT}$ and $\mathrm{MR}$ imaging are suggested due to the higher specificity (Fig. 2). Should an early fracture be suspected, fat-saturated MRI techniques (STIR or fatsat T2/proton density) are used to assess for bone marrow oedema of the stress response (Fig. 3). As the fracture is linear and located in the middle third of the bone, CT imaging must be performed perpendicular and parallel to the mid-

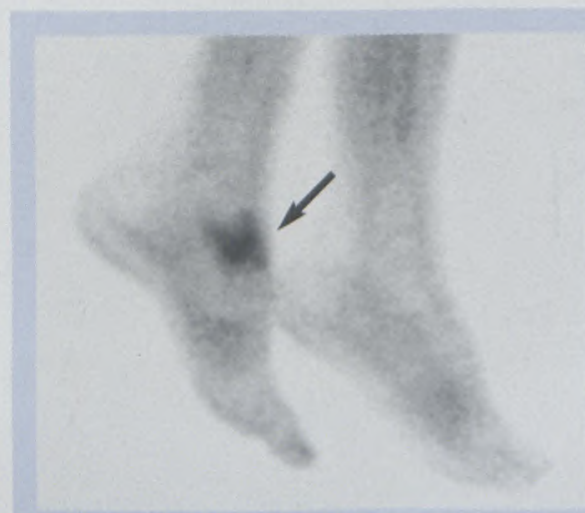

Fig. 2. Isotope bone scan of foot showing increased tracer uptake in navicular suggestive of a stress fracture.

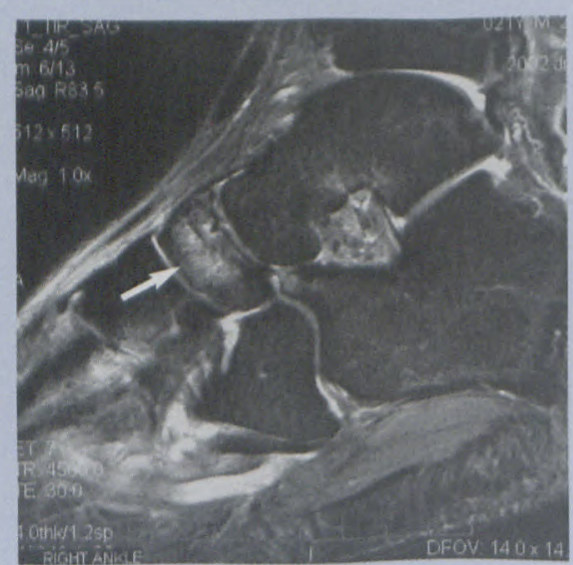

Fig. 3. MRI of the foot. Sagittal STIR image. Note increased signal intensity in the navicular with asso ciated fracture line compatible with occult fracture. No fracture was present on the plain X-ray. 


\section{CASE REPORT}

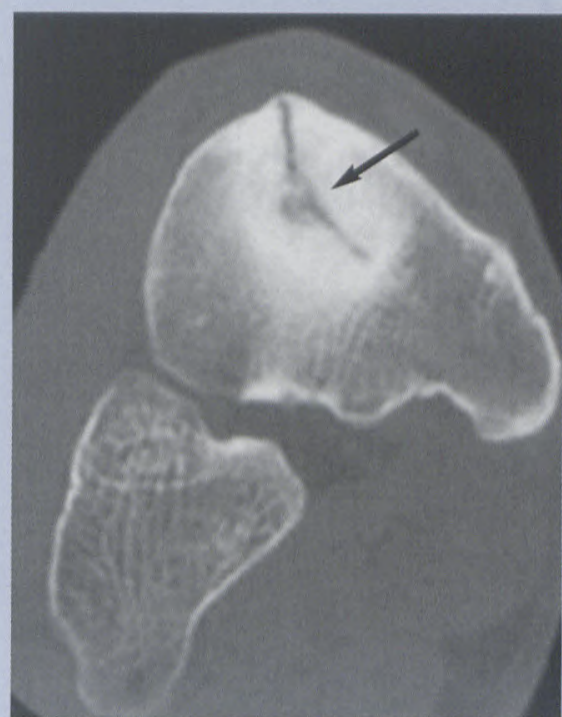

Fig. 4. Coronal CT of navicular. Note fracture line surrounded by sclerosis

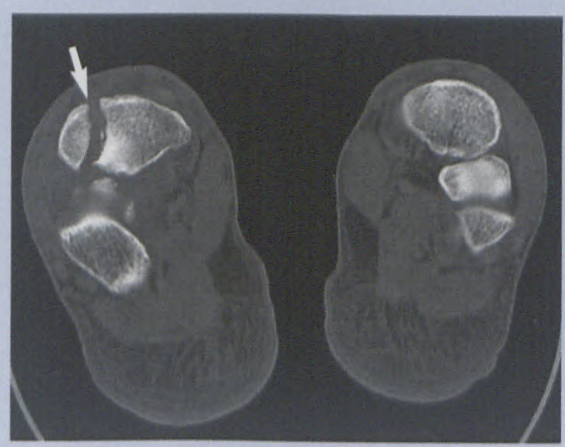

Fig. 5. Coronal CT of both navicula. Stress fracture of right navicular with complete fracture and displacement.

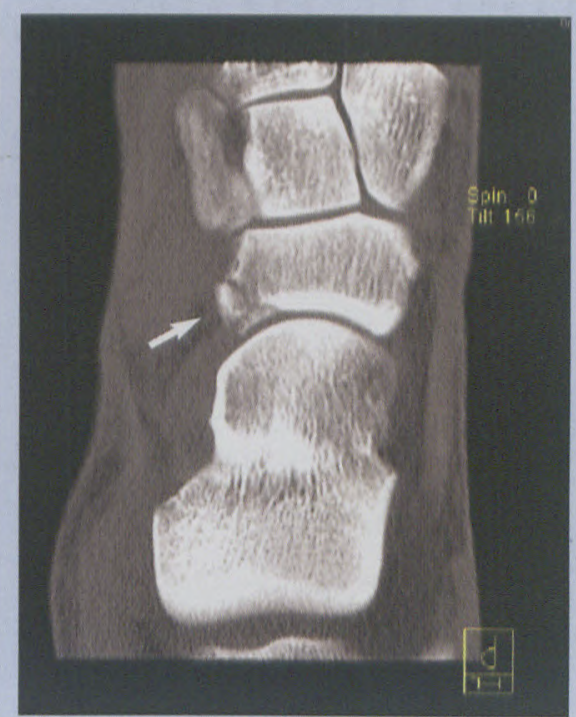

Fig. 6. Axial CT multiplanar image chip fracture of navicular.

foot (Fig. 4). Imaging of both feet allows internal comparison and may also detect an asymptomatic or unsuspected contralateral fracture (Fig 5). Multislice CT with multiplanar reformatting is the method of choice (Fig. 6). CT is a reliable indicator of fracture healing. The imaging evidence of fracture healing usually lags behind the clinical picture. ${ }^{3}$

\section{Treatment}

Navicular stress fractures are treated with cast immobilisation. This treatment results in a successful outcome in $80 \%$ of patients and most athletes return to sport in $5-6$ months. ${ }^{4}$

\section{References}

1. Georgen T, Venn-Watson E, Rossman D, et al. Tarsal navicular stress fractures in runners. $A J R$ 1981; 136: 339-346.

2. Pavlov H, Torg J, Freiberger R. Tarsal navicular stress fractures: radiographic evaluation. Radiology 1983; 148: 641-645.

3. Spitz DJ, Newberg AH. Imaging of stress fractures in the athlete. Radiol Clin North Am 2002; 40:324-326.

4. Khan K, Fuller P, Bruckner P. Outcome of conservative and surgical management of navicular stress fractures in athletes. Am J Sports Med 1992; 20: 657-666. 